\title{
Pour une Société Francaise de Physique influente et ouverte
}

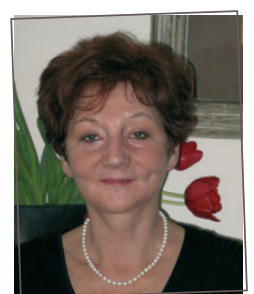

Je me sens très honorée de présider à mon tour, après tant de chercheurs brillants, aux destinées de la Société Française de Physique et je tiens à en remercier tous les membres pour leur confiance.

L'Année Mondiale de la Physique en 2005 m'a rapprochée de la SFP. Ce fut une extraordinaire aventure, avec l'imagination au pouvoir et les jeunes aux commandes et j'ai été très frappée du dynamisme des sections locales de la SFP. J'ai eu la très grande chance de travailler au côté de Roger Maynard et je pense continuer à faire équipe avec lui. La création de la revue Reflets de la physique et le renouvellement du site web, ont été les préalables au programme de la SFP aujourd'hui.

2007 est une année électorale qui accueille quantité de débats où, plus que jamais, la recherche et l'innovation ont une place importante. Le mouvement "Sauvons la Recherche ", puis les Assises de Grenoble en 2005, ont commencé à faire bouger l'opinion. Un train de mesures gouvernementales a suivi. Sont-elles adaptées ? Suffit-il d'augmenter les budgets de la recherche pour la rendre plus efficace et plus innovante ? Quels sont les vrais dangers des nanotechnologies ? Comment concilier la sortie du nucléaire à court terme avec la réduction du $\mathrm{CO}_{2}$ ? La gouvernance de nos universités est-elle compatible avec les défis du contexte mondial ?

Pour être entendue des politiques, la SFP doit d'abord se renforcer considérablement et s'ouvrir à de nouvelles catégories d'adhérents. Nous redémarrons une campagne en direction des jeunes - lycéens, étudiants, élèves des grandes écoles, doctorants, postdoctorants, enseignants et jeunes chercheurs. La SFP peut leur proposer des informations utiles pour leur orientation et leur carrière, un forum de discussion sur son site, des contacts à travers ses réseaux dans le monde professionnel, des débats sur les problèmes de société. Nous leur offrons une promotion à $10 €$ pour une adhésion d'un an, comportant Reflets. Espérons que cette campagne sera bien relayée par chacun de nous auprès des jeunes qui nous entourent.

C'est aussi avec nos collègues enseignants que la SFP souhaite renforcer ses liens. Ils sont les seuls à pouvoir redonner aux jeunes le goût des sciences de base. La revue Reflets fera un large écho aux préoccupations des professeurs et leur fournira des informations sur la physique actuelle, utilisables dans leurs classes. Un rapprochement avec l'Union des professeurs de spéciales s'est d'ailleurs amorcé (voir page 4). Et la commission " enseignement" de la SFP prend un nouveau départ avec une équipe constituée autour de Sylvie Magnier, Jean Cosleou et Arnaud Le Padellec. Elle souhaite être une force de proposition pour suivre et accompagner les évolutions de l'enseignement de la physique, tant sur les programmes que sur la pédagogie et les débouchés.

Le rapprochement avec les autres sociétés impliquant les sciences physiques en France est une autre nécessité, si l'on vise une audience dépassant le petit club des adhérents de chacune. La situation actuelle de complet morcellement est un héritage du passé. Beaucoup d'ingénieurs en France ne se considèrent même pas comme des physiciens. Un pas vers l'union a résulté de la création du G2P (Groupement d'action pour la Physique), qui s'est lancé dans une action pour faire connaître les métiers de la physique auprès des jeunes. Dans le but d'amorcer un rapprochement plus général et un jour plus visible, la SFP a commencé des discussions bilatérales avec plusieurs sociétés savantes. Diverses formules de partenariat et d'actions concrètes à mener en commun sont à l'étude. De plus, nous prenons des conseils pour mieux promouvoir notre société dans le monde industriel, susciter du mécénat et plus généralement, élargir notre audience.

Les autres chantiers de la SFP : énergie et environnement, coopération Nord-Sud, vous seront bientôt présentés. En outre, nous suivrons de très près, avec notre filiale EDP-Sciences, le préoccupant dossier des publications européennes dont les enjeux culturels et financiers sont importants.

L'année 2007 va être, avant tout, marquée pour la SFP par son congrès général en juillet à Grenoble. Le programme est passionnant. J'espère que vous serez très nombreux à avoir envie d'y assister et que vous aurez à cœur d'y attirer les jeunes.

Michèle Leduc 